\title{
DUALIZING COMPLEXES OF AFFINE SEMIGROUP RINGS
}

\author{
UWE SCHÄFER AND PETER SCHENZEL
}

\begin{abstract}
For an affine semigroup ring we construct the dualizing complex in terms of the semigroup and the homology of the face lattice of the polyhedral cone spanned by the semigroup. As a consequence there are characterizations of locally Cohen-Macaulay rings, Buchsbaum rings, and Cohen-Macaulay rings as well as Serre's condition $\mathscr{P}_{l}$.
\end{abstract}

\section{INTRODUCTION}

Let $S$ denote a finitely generated submonoid of the additive monoid $\mathbf{N}^{n}, n$ being a positive integer. By the affine semigroup ring $k[S]$ of $S$ over a field $k$ let us denote the subring of $k\left[x_{1}, \ldots, x_{n}\right]$ generated by all monomials $\underline{x}^{\underline{s}}$, $\underline{s} \in S$. So one has, up to isomorphisms, a one-to-one correspondence between affine semigroup rings and affine varieties given parametrically by monomials. Now it is of some interest to characterize ring theoretic properties as CohenMacaulay, Gorenstein, etc., in terms of the semigroup $S$. A first breakthrough in this direction was done by Kempf et al. [KKMS] and Hochster [Hol] who showed that $k[S]$ is a Cohen-Macaulay ring provided it is normal. Moreover, the normality of $k[S]$ is described in terms of $S$ (see [Ho1]).

Another particular case, if $S$ is a simplicial semigroup, is treated by Goto, Suzuki, and Watanabe [GSW], resp. Stanley [St2], who showed that $k[S]$ is a Cohen-Macaulay ring if it satisfies the $\mathscr{S}_{2}$-condition of Serre. Note that normality is equivalent to $\mathscr{S}_{2}$ and $R_{1}$. Moreover, there is a description of the $\mathscr{S}_{2}$-condition in terms of $S$ (see Theorem 6.3). In general the $\mathscr{S}_{2}$-condition is not sufficient for $k[S]$ to be a Cohen-Macaulay ring, as follows by examples in [Hol] resp. [TH, 1.2].

On the other side Gröbner [G] posed the problem to classify Cohen-Macaulay affine semigroup rings. The solution of this problem was done by Hoa and Trung [TH].

Related to structural properties of graded rings is a technical tool of the duality theory. This is the dualizing complex introduced by Grothendieck (see [Ha]). In recent years it became a helpful tool in commutative algebra. In particular, its knowledge allows characterizations of Cohen-Macaulay rings, locally CohenMacaulay rings, Buchsbaum rings, Gorenstein rings, and the $\mathscr{S}_{1}$-conditions of

Received by the editors December 18, 1988. 06A99.

1980 Mathematics Subject Classification (1985 Revision). Primary 13H10; Secondary 14M05, 
Serre. In general, the construction of the dualizing complex is not easy to describe in terms of the ring.

The main result of the present paper is the construction of $\mathscr{D}^{\circ}(\ell[S])$, the normalized dualizing complex of $k[S]$ in the category of $\mathbf{Z}^{n}$-graded modules (see Theorem 4.6). It is completely described in terms of the semigroup $S$ and the face lattice $\mathscr{F}_{S}$ of the convex rational polyhedral cone spanned by $S$ in $\mathbf{Q}^{n}$. The dualizing complex $\mathscr{D}^{*}(\mathscr{R}[S])$ provides a simplification of the CohenMacaulay criterion given by Hoa and Trung [TH] (see Theorem 6.13). Besides it there are characterizations of (1) the Serre condition $\mathscr{S}_{1},(2)$ the locally CohenMacaulayness of $k[S]$ and (3) to be a Buchsbaum ring, all in terms of $S$ and the face lattice.

In particular, the results about Buchsbaum rings, an intensive studied generalization of the Cohen-Macaulay rings (see [SV]), shed some more light on the interplay of commutative algebra, homological methods, and combinatorics.

Another description of the dualizing complexes of affine semigroup rings is given by Masa-Nori Ishida in [12] based on his work in [I1].

This paper is divided into six sections. The first section is concerned with basic constructions related to the semigroup $S$. Furthermore, it contains the results about the face lattice $F_{S}$. In the second section we recall basic work of Folkman [F] about the homology of lattices. The third section is concerned with basic facts about $\mathbf{Z}^{n}$-graded rings and modules, as well as the $\mathbf{Z}^{n}$-graded structure of $k[S]$. In Theorem 3.2, there is a description of the finite lattice of homogeneous prime ideals of $k[S]$. The aim of the fourth section, the core of the paper, is the construction of the dualizing complex of $k[S]$. Some of these techniques are used in the fifth section in order to calculate the local cohomology modules of $k[S]$ and the local cohomology modules of the canonical module of $\ell[S]$. The sixth section is devoted to applications and examples. We start with a characterization of Serre's $\mathscr{S}_{l}$-condition. Then there are criteria for $k[S]$ being a Cohen-Macaulay, a locally Cohen-Macaulay, and a Buchsbaum, resp. a Gorenstein, ring.

The question when $\ell[S]$ is a complete intersection ring, i.e., the defining ideal of $k[S]$ in $k\left[x_{1}, \ldots x_{n}\right]$ is generated by $n-d$ elements, $d=\operatorname{dim} k[S]$, is settled in [Sf].

\section{Standard SEMigroups}

First of all let us fix some notations, $\mathbf{N}, \mathbf{Q}\left(\mathbf{Q}^{+}\right), \mathbf{Z}$ denote the natural numbers $(0 \in \mathbf{N})$, the (positive) rationals, and the integers, respectively. Let $n$ denote a fixed positive integer. For the vector space $\mathbf{Q}^{n}$ we distinguish a base. Let $\underline{q}=\left(q_{1}, \ldots, q_{n}\right)$ denote the coordinates of $\underline{q} \in \mathbf{Q}^{n}$ with respect to this distinguished base. For $v \in \mathbf{Q}, V \subseteq \mathbf{Q}, \mathscr{W}, \mathscr{W}^{\prime} \subseteq \mathbf{Q}^{n}$ put

$$
\begin{gathered}
\mathscr{W} \pm \mathscr{W}^{\prime}=\left\{\underline{w} \pm \underline{w}^{\prime} \mid \underline{w} \in \mathscr{W}, \underline{w}^{\prime} \in \mathscr{W}^{\prime}\right\}, \\
v \mathscr{W}=\{v \cdot \underline{w} \mid \underline{w} \in \mathscr{W}\}, \quad V \mathscr{W}=\{v \cdot \underline{w} \mid v \in V, \underline{w} \in \mathscr{W}\} .
\end{gathered}
$$


By " $\leq$ " let us denote the partial order on $\mathbf{Q}^{n}$ induced by the order of the components. An affine semigroup $S$ is defined as a finitely generated submonoid of the monoid $\mathbf{N}^{n}$ with the componentwise addition and $\underline{0}=(0, \ldots, 0)$ as the neutral element. $G(S)$ denotes the group generated by $S$ in $\mathbf{Z}^{n}$. The monoid

$$
\bar{S}=\{\underline{s} \in G(S) \mid \text { there is an } m \in \mathbf{N} \backslash\{0\} \text { with } m \underline{s} \in S\}
$$

is called the normalization of $S$.

For a subset $U$ of $\mathbf{N}^{n}$, denote by $\langle U\rangle$ the monoid generated by the elements of $U$. In particular, $\langle\varnothing\rangle=\{\underline{0}\}$. Let $E(S)$ denote the set of indecomposable elements (with respect to the addition) of $S \backslash\{\underline{0}\}$. Then $E(S)$ is the uniquely determined minimal generating system of $S$.

1.1. Lemma. For an arbitrary subset $U$ of $S,\langle U\rangle=S$ if and only if $U \supseteq E(S)$. Proof. Obviously $\langle E(S)\rangle=S$. Then the claim follows because of $\underline{u} \notin\langle U\rangle$ for $\underline{u} \in E(S) \backslash U$.

$\mathscr{C}_{S}:=\mathbf{Q}^{+} S$ is a convex polyhedral cone in the linear subspace $\mathbf{Q} S$ of $\mathbf{Q}^{n}$ with the vertex $\underline{0}$ and the dimension rank $G(S)$. The minimal elements of $S \backslash\{\underline{0}\}$ on the edges of this cone form a subset $E_{0}(S)$ of $E(S)$ with the following properties.

1.2. Lemma. (a) $\mathscr{C}_{S}=\mathbf{Q}^{+}\left\langle E_{0}(S)\right\rangle$. (b) There is a positive integer $h$ such that $h S \subseteq\left\langle E_{0}(S)\right\rangle$.

Proof. The first claim follows because $\mathscr{C}_{S}$ is the convex hull of its edges. The second part is obvious, now.

Put $d:=\operatorname{rank} G(S)$. The following is well known (cf. [Br, §2]): The nonempty faces of $\mathscr{C}_{S}$ are convex polyhedral cones with vertex $\underline{0}$. With respect to inclusion, they form a finite lattice $F_{S}$ graded by the dimension of its elements. We have $\left(F_{S}\right)_{0}=\{\{\underline{0}\}\},\left(F_{S}\right)_{d}=\left\{\mathscr{C}_{S}\right\}$. Any maximal totally ordered subset of $F_{S}$ consists of exactly $d+1$ elements. For $0 \leq i<j<d$, any element of $\left(F_{S}\right)_{i}$ is the intersection of elements of $\left(F_{S}\right)_{j}$.

1.3. Definition. An affine semigroup $S$ is called a standard semigroup provided the following conditions are satisfied.

(1) $\bar{S}=G(S) \cap \mathbf{N}^{n}$.

(2) For $i=1,2, \ldots, n$, the image of $S$ by the projection $\pi_{i}$ on the $i$ th component is a numerical semigroup, i.e., $\mathbf{N} \backslash \pi_{i}(S)$ is a finite set.

(3) The semigroups $F_{i}=S \cap \operatorname{ker} \pi_{i}, i=1,2, \ldots, n$, are pairwise disjoint with $\operatorname{rank} G\left(F_{i}\right)=\operatorname{rank} G(S)-1$.

By Hochster's result [Hol, $\S 2]$, for any semigroup there is an isomorphic standard semigroup. In the following let us assume $S$ to be a standard semigroup. The conditions (1) and (3) mean that

$$
\left(F_{S}\right)_{d-1}=\left\{\mathbf{Q}^{+} F_{i} \mid i=1,2, \ldots, n\right\} .
$$


In particular we see that $\mathscr{C}_{S}$ has exactly $n$ maximal proper faces. All faces of $\mathscr{C}_{S}$ admit a characterization in terms of the vanishing of certain components. More precisely, for a subset $I$ of $\{1,2, \ldots, n\}$ let

$$
F_{I}=\left\{\underline{s} \in S \mid s_{j}=0 \text { for } j \in I\right\} .
$$

For the case $I=\{j\}$, this means $F_{\{j\}}=F_{j}$ as defined in Definition 1.3.

1.4. Lemma. (a) $F_{S}=\left\{\mathbf{Q}^{+} F_{I} \mid I \subseteq\{1,2, \ldots, n\}\right\}$. (b) For any subset $I$ of $\{1,2, \ldots, n\}$ there is a unique maximal subset $r(I)$ of $\{1,2, \ldots, n\}$ such that $F_{I}=F_{r(I)}$.

The proof follows easily because $F_{S}$ is closed with respect to intersections. Let $\operatorname{Max}_{S}$ denote the set of maximal subsets $I$ (with respect to inclusions) of $\{1,2, \ldots, n\}$ with $r(I) \neq\{1,2, \ldots, n\}$. Then $r(I)=I$ for every $I \in \operatorname{Max}_{S}$ by Lemma 1.4. Furthermore, we have

\subsection{Lemma. $\left(F_{S}\right)_{1}=\left\{\mathbf{Q}^{+} F_{l} \mid I \in \operatorname{Max}_{S}\right\}$.}

Proof. The inclusion $\left(F_{S}\right)_{1} \subseteq\left\{\mathbf{Q}^{+} F_{I} \mid I \in \operatorname{Max}_{S}\right\}$ follows by the definition of $\operatorname{Max}_{S}$. Conversely let $I \in \operatorname{Max}_{S}$. Suppose $\operatorname{dim} \mathbf{Q}^{+} F_{I}>1$. Let $\underline{s}, \underline{s^{\prime}} \in F_{I}$ be linearly independent over $\mathbf{Q}$. Then there exists an $s^{\prime \prime} \in \mathbf{N}^{n}$ such that $\underline{0} \neq \underline{s}^{\prime \prime}=h \underline{s}+h^{\prime} \underline{s}^{\prime}, h, h^{\prime} \in \mathbf{Z}$, and $I \subset\left\{i \mid s_{i}^{\prime \prime}=0\right\}$. But $\underline{s}^{\prime \prime} \in G(S) \cap \mathbf{N}^{n}=\bar{S}$. Thus we have a contradiction to the maximality of $I$. Therefore $\mathbf{Q}^{+} F_{I} \in\left(F_{S}\right)_{1}$ as required.

1.6. Remark. By virtue of 1.5

$$
E_{0}(S) \ni \underline{s} \mapsto\left\{i \mid s_{i}=0\right\}
$$

yields a one-to-one correspondence between $E_{0}(S)$ and $\operatorname{Max}_{S}$.

Example. For $d \leq 3$ there is a complete classification (up to isomorphisms) of the sets $\operatorname{Max}_{S}$ :

(i) $\operatorname{Max}_{S}=\{\varnothing\}$ and $n=1$, for $d=1$.

(ii) $\operatorname{Max}_{S}=\{\{1\},\{2\}\}$ and $n=2$, for $d=2$.

(iii) $\operatorname{Max}_{S}=\{\{1,2\},\{2,3\}, \ldots,\{n-1, n\},\{n, 1\}\}$ for $d=3$ and an $n \geq 3$.

This follows easily by virtue of known geometric results.

In the next part we summarize identities for certain difference sets, which is important for the fine study of semigroup rings.

1.7. Theorem. Let $s \in S$ and $I=\left\{i \mid s_{i}=0\right\}$. Then $S-\langle\{\underline{s}\}\rangle=S-F_{I}$.

Proof. Because of $\underline{s} \in F_{I}$ we have $S-\langle\{\underline{s}\}\rangle \subseteq S-F_{I}$. For the reverse inclusion, let $\underline{t} \in S$ and $\underline{t}^{\prime} \in F_{I}$. Then there are positive integers $g, g^{\prime}$ such that $\underline{g} \underline{s} \geq \underline{t}^{\prime}$ i.e., $\underline{g} \underline{s}-\underline{t}^{\prime} \in \bar{S}$, and $g^{\prime}\left(g \underline{s}-\underline{t}^{\prime}\right) \in S$. Therefore

$$
\underline{t}-\underline{t}^{\prime}=\left(\underline{t}+\left(g^{\prime}-1\right) \underline{t^{\prime}}+g^{\prime}\left(\underline{g} \underline{s}-\underline{t}^{\prime}\right)\right)-g g^{\prime} \underline{s} \in S-\langle\{\underline{s}\}\rangle
$$

as required. 
Now we decompose the group $G(S) \subseteq \mathbf{Z}^{n}$ into a disjoint union of certain subsets. To this end let $\mathscr{U}$ denote a filter on $F_{S}$, i.e., a proper nonempty subset of the lattice $F_{S}$ which contains with a face all faces containing it. Put

$$
S_{\mathscr{U}}=\bigcap_{\mathbf{Q}^{+} F_{I} \in \mathscr{U}}\left(S-F_{I}\right) \backslash \bigcup_{\mathbf{Q}^{+} F_{J} \notin \mathscr{U}}\left(S-F_{J}\right)
$$

For different filters $\mathscr{U}, \mathscr{U}^{\prime}$ follows $S_{\mathscr{U}} \cap S_{\mathscr{U}^{\prime}}=\varnothing$ because of the construction. Hence, $S_{\mathscr{U}}$ yields the requested disjoint union of $G(S)$ when $\mathscr{U}$ covers all filters of $F_{S}$. For particular cases of standard semigroups, there is a more simple description of this family of sets. To this end put

$$
S^{\prime}=\bigcap_{i=1}^{n} S-F_{i} .
$$

Then $S^{\prime}$ is again a standard semigroup with $S \subseteq S^{\prime} \subseteq \bar{S}$.

1.8. Lemma. (a) $S^{\prime}-F_{I}=\bigcap_{i \in r(I)} S^{\prime}-F_{i}=\bigcap_{i \in r(I)} S-F_{i}$ for every nonempty subset $I$ of $\{1, \ldots, n\}$.

(b) If $S_{\mathscr{U}}^{\prime} \neq \varnothing$, then $\mathscr{U}=\mathscr{U}(J)=\left\{\mathbf{Q}^{+} F_{I} \mid I \subseteq J\right\}$ for some subset $J$ of $\{1, \ldots, n\}$.

Proof. The first statement is shown by Goto and Watanabe [GW2, Lemma 3.3.5]. The second claim follows because of

$$
S_{\mathscr{U}}^{\prime}=\bigcap_{\mathbf{Q}^{+} F_{i} \in \mathscr{U}}\left(S-F_{i}\right) \backslash \bigcup_{\mathbf{Q}^{+} F_{j} \notin \mathscr{U}}\left(S-F_{j}\right) .
$$

In the following let us continue with the investigation of $S_{\mathscr{U}(J)}^{\prime}$.

1.9. Lemma. Let $S_{\mathscr{U}(J)}^{\prime} \neq \varnothing$ for some $J \subseteq\{1, \ldots, n\}$.

(a) If $S_{\mathscr{U}(J)}^{\prime}$ is bounded above, then $r(J)=\{1, \ldots, n\}$.

(b) If $S_{\mathscr{U}(J)}^{\prime}$ is bounded below, then $r(\{1, \ldots, n\} \backslash J)=\{1, \ldots, n\}$.

(c) Let $r(J)=r(\{1, \ldots n\} \backslash J)$ and $S_{\mathscr{U}(J)}^{\prime}$ bounded above (resp. below).

Then it is bounded below (resp. above).

(d) In the case $\varnothing \neq J=r(J), S_{\mathscr{U}(J)}^{\prime}$ is not bounded above.

Proof. It follows easily that

$$
\begin{aligned}
& S_{\mathscr{U}(J)}^{\prime}-F_{r(J)} \subseteq S_{\mathscr{U}(J)}^{\prime} \quad \text { and } \\
& S_{\mathscr{U}(J)}^{\prime}+F_{r(\{1, \ldots, n\} \backslash J)} \subseteq S_{\mathscr{U}(J)}^{\prime} .
\end{aligned}
$$

This proves (a) and (b). In order to show (c), let $\underline{t} \in \mathbf{Z}^{n}$ be an upper bound of $S_{\mathscr{U}(J)}^{\prime}$. Hence $0 \leq s_{i} \leq t_{i}$ for all $s \in S_{\mathscr{U}(J)}$ and $i \in J$. Therefore, $S_{\mathscr{U}(J)}^{\prime}$ is the union of the finitely many sets

$$
S_{\mathscr{U}(J)}^{\prime}(\underline{s})=\left\{\underline{u} \in S_{\mathscr{U}(J)} \mid u_{i}=s_{i}, i \in J\right\} .
$$


In the case $S_{\mathscr{U}(J)}^{\prime}$ is an infinite set, the same holds for one of the sets $S_{\mathscr{U}(J)}^{\prime}(\underline{s})$, $\underline{s} \in S_{\mathscr{U}(J)}^{\prime}$. But then there are two different elements $\underline{s}^{\prime}, \underline{s}^{\prime \prime} \in S_{\mathscr{U}(J)}^{\prime}(\underline{s})$, such that $\underline{s}^{\prime} \geq \underline{s}^{\prime \prime}$. It follows $J \subseteq J^{\prime}=r\left(J^{\prime}\right) \neq\{1, \ldots, n\}$ for $J^{\prime}=\left\{i \in\{1, \ldots, n\} \mid s_{i}^{\prime}=\right.$ $\left.s_{i}^{\prime \prime}\right\}$. Whence $r(J) \neq\{1, \ldots, n\}$, a contradiction. The second case follows the same line of reasoning. For the proof of (d) consider $\underline{s} \in S^{\prime}-F_{J}$ such that $s_{i}<0$, if $i \notin J$, and $s_{j}>0$ for at least one $j$. Such an $\underline{s}$ exists because $J \neq \varnothing$. But then, $h \underline{s} \in S_{\mathscr{U}(J)}^{\prime}$ for $h=1,2,3, \ldots$. Whence $S_{\mathscr{U}(J)}^{\prime}$ is not bounded above.

The importance of those boundedness conditions is closely related to the following situation. Let $N \subseteq G(S)$ such that $N+S \subseteq N$.

1.10. Lemma. There exist finitely many elements $\underline{t}^{1}, \underline{t}^{2}, \ldots, \underline{t}^{\prime} \in G(S)$ such that $N=\left\{\underline{t}^{1}, \underline{t}^{2}, \ldots, \underline{t}^{l}\right\}+S$ if and only if $N$ is bounded below.

Proof. In the case there exist elements $t^{1}, \ldots, t^{l}$ with the desired property and the infimum of these elements yields a lower bound of $N$. In a first step we prove the reverse conclusion for $N=\bar{S}$. By Dickson's lemma [Di], a lower bounded subset of $\mathbf{Z}^{n}$ has only a finite number of minimal elements. Applied to $\bar{S} \backslash\{\underline{0}\}$, this yields that it is a finitely generated monoid. Let $E(\bar{S})=$ $\left\{\underline{s}^{1}, \ldots, \underline{s}^{g}\right\}$. Then there is a positive integer $m$ such that $m \underline{s}^{i} \in S$ for all $i=1, \ldots, g$. Therefore

$$
\bar{S}=\left\{\sum_{i=1}^{g} m_{i} \underline{s}^{i} \mid m_{i} \in \mathbf{N}, m_{i}<m\right\}+S .
$$

For an arbitrary lower bounded subset $N$ of $G(S)$, it follows that $\operatorname{Min}(N)$, the set of minimal elements of $N$, is finite. Because of $N \subseteq \operatorname{Min}(N)+\bar{S}$, it yields

$$
N \subseteq\left(\operatorname{Min}(N)+\left\{\sum_{i=1}^{g} m_{i} \underline{s}^{i} \mid m_{i} \in \mathbf{N}, m_{i}<m\right\}\right)+S .
$$

This proves the claim.

1.11. Example. An affine standard semigroup ring is called simplicial provided

$$
\operatorname{Max}_{S}=\{\{1, \ldots, n\} \backslash\{i\} \mid i=1, \ldots, n\} .
$$

This is equivalent to the fact that $E_{0}(S)$ consists of exactly $d$ elements (cf. [TH]). Of Course $d=n$. Then every subset $J$ of $\{1, \ldots, n\}$ has the property $J=r(J)$. By Lemma 1.8(d), all of the sets $S_{1 /(J)}^{\prime}, J \neq \varnothing$, are either empty or not bounded above.

\section{Homology GROUPS OF A Finite LATTICE}

Homological investigations of affine semigroup rings lead to investigations on the lattice $\mathscr{F}_{S}$. To make these connections more precise, let us fix the required definitions. Let $\mathscr{V}$ denote a finite lattice with $\overline{0}$ the infimum and 
$\overline{1}$ the supremum of $\mathscr{V}$. A totally ordered subset of $\mathscr{V} \backslash\{\overline{0}, \overline{1}\}$ with $m+1$ elements is called a chain of length $m$. The order complex of $\mathscr{V}$ is defined as the abstract simplicial complex whose $m$-dimensional faces are the chains of length $m$ of $\mathscr{V}$. The homology groups $H_{i}(\mathscr{V} ; G)$ of $\mathscr{V}$ with coefficients in an abelian group $G$ are defined as the corresponding reduced homology groups of the order complex.

A subset $\mathscr{V}^{0}$ of $\mathscr{V} \backslash\{\overline{0}, \overline{1}\}$ is called a crosscut of $\mathscr{V}$ if the elements of $\mathscr{V}^{0}$ are pairwise incomparable and every maximal chain of $\mathscr{V}$ contains an element of $\mathscr{V}^{0}$. Let $\Delta\left(\mathscr{V}^{0}\right)$ denote the abstract simplicial complex of all subsets $\mathscr{W}$ of $\mathscr{V}^{0}$ with

$$
\{\inf \mathscr{W}, \sup \mathscr{W}\} \neq\{\overline{0}, \overline{1}\} .
$$

Then the following theorem was shown by Folkman [F].

2.1. Theorem [F]. Let $\mathscr{V}^{0}$ be a crosscut of $\mathscr{V}$. Then

$$
H_{i}(\mathscr{V} ; G)=\widetilde{H}_{i}\left(\Delta\left(\mathscr{V}^{0}\right) ; G\right)
$$

for any $i \in \mathbf{Z}$ and an abelian group $G$.

We will apply this result to quotients of the lattice $\mathscr{F}_{S}$. To this end, let $\mathscr{U}$ be a filter of $\mathscr{F}_{S}$. Let $\mathscr{F}_{S} / \mathscr{U}$ denote the factor lattice of $\mathscr{F}_{S}$ with respect to $\mathscr{U}$. Define $\operatorname{Max}_{S}=\left\{J_{1}, \ldots, J_{r}\right\}$ as in the first section. Let $\Delta_{\mathscr{U}}$ denote the subcomplex of $\Delta$, the complex of all subsets of $\{1, \ldots, r\}$, defined by $\alpha \in$ $\Delta_{\mathscr{U}}$ if and only if $\mathbf{Q}^{+} F_{\cap_{i \in \alpha}} J_{i} \notin \mathscr{U}$. Then $\left\{\mathbf{Q}^{+} F_{J} \mid J \in \operatorname{Max}_{S}\right\} \backslash \mathscr{U}$ is a crosscut of $\mathscr{F}_{S} / \mathscr{U}$. Therefore Theorem 2.1 yields the following result.

2.2. Corollary. $H_{i}\left(\mathscr{F}_{S} / \mathscr{U} ; G\right)=\widetilde{H}_{i}\left(\Delta_{\mathscr{U}} ; G\right)$ for any $i \in \mathbf{Z}$ and an abelian group $G$.

Let us continue with some examples.

2.3. Examples. (a) Let $\Delta$ be a simplicial complex with the vertex set $E$. Then $\bar{\Delta}=\Delta \cup\{\varnothing, E\}$ is a finite lattice. The order complex of $\bar{\Delta}$ is the barycentric subdivision of $\Delta$. Hence

$$
H_{i}(\bar{\Delta} ; G)=\widetilde{H}_{i}(\Delta ; G) .
$$

(b) The intersection of the cone $\mathscr{C}_{S}=\mathbf{Q}^{+} S$ with the hyperplane $\sum q_{i}=1$ in the linear space $\mathbf{Q} G(S)$ is a $(d-1)$-dimensional convex polyhedron with edge lattice $\mathscr{F}_{S}$. Whence we have

$$
H_{i}\left(\mathscr{F}_{S} ; G\right)= \begin{cases}G, & i=d-2 ; \\ 0, & \text { otherwise }\end{cases}
$$

\section{3. $\mathbf{N}^{n}$-GRADED RINGS}

A theory of $\mathbf{N}^{n}$-graded rings and modules was developed by Goto and Watanabe [GW2], resp. Stanley [St1]. Here we summarize the basic notions. 
3.1. Definition. A commutative ring $\mathscr{R}$ with 1 is called $\mathbf{N}^{n}$-graded provided there is a family $\left\{\mathscr{R}_{\underline{s}} \mid \underline{s} \in \mathbf{N}^{n}\right\}$ of subgroups of $\mathscr{R}$ such that $\mathscr{R}=\bigoplus_{\underline{s} \in \mathbf{N}^{n}} \mathscr{R}_{\underline{s}}$ and $\mathscr{R}_{\underline{s}} \mathscr{R}_{\underline{t}} \subseteq \mathscr{R}_{\underline{s}+\underline{t}}$ for $\underline{s}, \underline{t} \in \mathbf{N}^{n}$.

A module $\mathscr{M}$ over an $\mathbf{N}^{n}$-graded ring $\mathscr{R}$ is called $\mathbf{Z}^{n}$-graded if there is a family $\left\{\mathscr{M}_{\underline{\underline{s}}} \mid \underline{t} \in \mathbf{Z}^{n}\right\}$ of subgroups of $\mathscr{M}$ such that $\mathscr{M}=\bigoplus_{\underline{t} \in \mathbf{Z}^{n}} \mathscr{M}_{\underline{t}}$ and $\mathscr{R}_{\underline{s}} \mathscr{M}_{\underline{t}} \subseteq$ $\mathscr{M}_{\underline{s}+\underline{t}}$ for $\underline{\underline{s}} \in \mathbf{N}^{n}, \underline{t} \in \mathbf{Z}^{n}$.

The elements of $\mathscr{R}_{s}$ resp. $\mathscr{M}_{t}$ are called homogeneous of degree $\underline{s}$ resp. $\underline{t}$. An ideal of an $\mathbf{N}^{n}$-graded ring $\mathscr{R}$ is homogeneous provided it has a homogeneous generating set, i.e., it is a $\mathbf{Z}^{n}$-graded submodule of $\mathscr{R}$. An $\mathscr{R}$-linear map $\gamma: \mathscr{M} \rightarrow \mathbf{N}$ of $\mathbf{Z}^{n}$-graded $\mathscr{R}$-modules $\mathscr{M}$ and $\mathscr{N}$ is called a homomorphism provided $\gamma\left(\mathscr{M}_{\underline{t}}\right) \subseteq \mathscr{N}_{\underline{t}}, \underline{t} \in \mathbf{Z}^{n}$. For $\underline{u} \in \mathbf{Z}^{n}$ let $\mathscr{N}(\underline{u})$ denote the $\mathbf{Z}^{n}$-graded $\mathscr{R}$-module $\mathscr{N}^{-}$whose grading is given by

$$
\mathscr{N}(\underline{u})_{\underline{t}}=\mathcal{N}_{\underline{u}+\underline{t}}, \quad \underline{t} \in \mathbf{Z}^{n} .
$$

Let $\underline{\operatorname{Hom}}_{\mathscr{R}}(\mathscr{M}, \mathscr{N})_{\underline{u}}$ denote the group of homomorphisms of the $\mathbf{Z}^{n}$-graded $\mathscr{R}$-modules $\mathscr{M}$ and $\mathscr{N}(\underline{u})$. Then

$$
\underline{\operatorname{Hom}}_{\mathscr{A}}(\mathscr{M}, \mathscr{N})=\bigoplus_{\underline{u} \in \mathbf{Z}^{n}} \underline{\operatorname{Hom}}_{\mathscr{R}}(\mathscr{M}, \mathscr{N})_{\underline{u}}
$$

is a $\mathbf{Z}^{n}$-graded $\mathscr{R}$-module.

Now let $\mathscr{R}$ be a Noetherian $\mathbf{N}^{n}$-graded ring such that $\mathscr{R}_{0}$ is a local ring with the maximal ideal $m_{\underline{0}}$. Then $m=m_{0} \oplus \bigoplus_{\underline{s} \neq \underline{0}} \mathscr{R}_{\underline{s}}$ denotes the unique homogeneous maximal ideal of $\mathscr{R}$. Call $\mathscr{R}$ a Cohen-Macaulay, Gorenstein, resp. Buchsbaum ring provided $\left(\mathscr{R}_{m}, m \mathscr{R}_{m}\right)$ has the corresponding property.

Let $\rho$ denote a prime ideal and $\mathscr{M}$ a $\mathbf{Z}^{n}$-graded $\mathscr{R}$-module. Then $\mathscr{M}_{(\mu)}$ denotes the localization of $\mathscr{M}$ with respect to the multiplicatively closed set of homogeneous elements of $\mathscr{R} \backslash n$.

Let $S \subseteq \mathbf{N}^{n}$ denote a standard semigroup. Let $h$ be an arbitrary field. Let us write $\underline{x}^{\underline{s}}, \underline{s} \in \mathbf{N}^{n}$; for the monomial $x_{1}^{s_{1}} \cdots x_{n}^{s_{n}}$ in $\ell[\underline{x}]=\ell\left[x_{1}, \ldots, x_{n}\right]$, the polynomial ring in the indeterminates $x_{1}, \ldots, x_{n}$ over $k$. The subring $k[S]$ of $k[\underline{x}]$ generated by all the monomials $\underline{x}^{\underline{s}}, \underline{s} \in S$, is called the affine semigroup ring of $S . \not R[S]$ is an $\mathbf{N}^{n}$-graded ring because of

$$
k[S]_{\underline{s}}= \begin{cases}h, & \underline{s} \in S \\ 0, & \underline{s} \notin S .\end{cases}
$$

Let $N$ be a subset of $\mathbf{Z}^{n}$ with the property $N+S \subseteq N$. Then $k[N]$ denotes the $\mathbf{Z}^{n}$-graded $h[S]$-module defined in a similar way. The homogeneous maximal ideal of $h[S]$ is $m=k[S \backslash\{\underline{0}\}]$. For two subsets $N_{1}, N_{2}$ of $\mathbf{Z}^{n}$ with $N_{1} \supseteq N_{2}$ set

$$
\mathscr{k}\left[N_{1} \backslash N_{2}\right]=\mathscr{k}\left[N_{1}\right] / \mathscr{k}\left[N_{2}\right] .
$$

By Trung and Hoa [TH, Lemma 2.1], the homogeneous prime ideals of $k[S]$ are exactly given by $k\left[S \backslash F_{J}\right], J \subseteq\{1, \ldots, n\}$. Here we prove 
3.2. Theorem. (a) The homogeneous prime ideals of $\ell[S]$ form a lattice, antiisomorphic (with respect to inclusion) to $\mathscr{F}_{S}$.

(b) Let $J \subseteq\{1, \ldots, n\}$ such that $\mathbf{Q}^{+} F_{J} \in\left(\mathscr{F}_{S}\right)_{i}$ for some $i \in \mathbf{N}$. Then

$$
\operatorname{dim} \ell\left[F_{J}\right]=i \text { and } h t k\left[S \backslash F_{J}\right]=d-i,
$$

$d=\operatorname{rank} G(S)$.

Proof. For (a) note that the order-reversing bijection is given by $k\left[S \backslash F_{J}\right] \mapsto$ $\mathbf{Q}^{+} F_{J}$. Then (b) follows by properties of $\mathscr{F}_{S}$ shown in the first section. Now let $n_{J}=\ell\left[S \backslash F_{J}\right], J \subseteq\{1, \ldots, n\}$, a homogeneous prime ideal. Note that $\not S]_{\left(n_{J}\right)}=\not h\left[S-F_{J}\right]$.

The following result is useful for the next section in order to construct the dualizing complex.

3.3. Lemma. The set of monomials $\left\{\underline{x}^{\underline{s}} \mid \underline{s} \in E_{0}(S)\right\}$ generates an m-primary ideal of $k[S]$.

Proof. By $1: 2$ there exists an integer $h$ such that

$$
h(S \backslash\{\underline{0}\}) \subseteq\left\langle E_{0}(S)\right\rangle \subseteq E_{0}(S)+S .
$$

But this means that the ideal generated by the described set of monomials contains $m^{h}$. This proves the claim.

\section{DuALiZing COMPLEXES}

Now let $\mathscr{R}$ denote an $\mathbf{N}^{n}$-graded ring, with $\mathscr{R}_{0}=k$ a field. Put $m=$ $\bigoplus_{\underline{s} \neq \underline{0}} \mathscr{R}_{\underline{s}}$. Let $\underline{\vartheta} \mathscr{R}$ denote the homogeneous ideal generated by forms $\vartheta_{1}, \ldots$, $\vartheta_{r}$. The complex $\mathscr{K}^{*}\left(\vartheta_{i} ; \mathscr{R}\right), i=1, \ldots, r$, is defined by

$$
\mathscr{K}^{i}\left(\vartheta_{i} ; \mathscr{R}\right)= \begin{cases}\mathscr{R}, & i=0, \\ \mathscr{R}_{\vartheta_{i}}, & i=1, \\ 0, & \text { otherwise, }\end{cases}
$$

with the only nontrivial boundary homomorphism

$$
\mathscr{K}^{0}\left(\vartheta_{i} ; \mathscr{R}\right) \rightarrow \mathscr{K}^{1}\left(\vartheta_{i} ; \mathscr{R}\right), \quad r \mapsto \frac{r}{1} .
$$

Here $\mathscr{R}_{\vartheta}$ denotes the localization of $\mathscr{R}$ with respect to the multiplicatively closed set $\left\{\vartheta^{r}, r \in \mathbf{N}\right\}$. For a finitely generated $\mathbf{Z}^{n}$-graded $\mathscr{R}$-module $\mathscr{M}$ put

$$
\mathscr{K}^{\cdot}(\underline{\vartheta} ; \mathscr{M})=\left(\bigotimes_{i=1}^{r} \mathscr{K}^{\cdot}\left(\vartheta_{i} ; \mathscr{R}\right)\right) \otimes_{\mathscr{R}} \mathscr{M} .
$$

This is a complex of $\mathbf{Z}^{n}$-graded $\mathscr{R}$-modules with

$$
H^{i}\left(\mathscr{K}^{\cdot}(\underline{\vartheta} ; \mathscr{M})\right) \cong H_{\underline{\vartheta}}^{i}(\mathscr{M}), \quad i \in \mathbf{Z},
$$


where $H_{\vartheta}^{i}(\mathscr{M}), i \in \mathbf{Z}$, denotes the local cohomology modules of $\mathscr{M}$ with support $\underline{\vartheta} \mathscr{R}$. See $[\mathrm{Gr}]$ for the definitions and the details of the proofs. In particular, it follows:

1. $H_{\underline{\vartheta}}^{i}(\mathscr{M}), i \in \mathbf{Z}$, are $\mathbf{Z}^{n}$-graded Artinian $\mathscr{R}$-modules, if $\operatorname{Rad} \underline{\vartheta} \mathscr{R}=m$.

2. Up to isomorphism $\mathscr{K}^{*}(\underline{\vartheta} ; \mathscr{R})$ does not depend on the particular choice of the generating set.

Let $\mathscr{M}$ denote a $\mathbf{Z}^{n}$-graded $\mathscr{R}$-module. For a complex $\mathscr{C}^{\cdot}$ of $\mathbf{Z}^{n}$-graded $\mathscr{R}$-modules the complex $\underline{\mathrm{Hom}}_{\mathscr{A}}\left(\mathscr{C}^{\circ}, \mathscr{M}\right)$ is defined by

$$
\left(\underline{\operatorname{Hom}}_{\mathscr{A}}\left(\mathscr{C}^{\cdot}, \mathscr{M}\right)\right)^{i}=\underline{\operatorname{Hom}}_{\mathscr{A}}\left(\mathscr{C}^{-i}, \mathscr{M}\right), \quad i \in \mathbf{Z},
$$

and the induced boundary maps. For an integer $p$ denote by $\mathscr{M}[p]$ the complex

$$
\mathscr{M}[p]^{i}= \begin{cases}\mathscr{M}, & i=p, \\ 0, & i \neq p,\end{cases}
$$

with trivial boundary maps. For the definition of $\mathbf{Z}^{n}$-graded injective $\mathscr{R}$ modules see [GW2]. In accordance with Hartshorne [Ha], we define

4.1. Definition. A dualizing complex of $\mathscr{R}$ is a bounded complex $\mathscr{D}^{\circ}(\mathscr{R})$ of $\mathbf{Z}^{n}$-graded injective $\mathscr{R}$-modules and homomorphisms whose cohomology modules are finitely generated and $\underline{\operatorname{Hom}}_{\mathscr{R}}\left(h, \mathscr{D}^{\circ}(\mathscr{R})\right)=h[p]$ for an integer $p$. Here $h$ is considered as the $\mathscr{R}$-module $\mathscr{R} \mid \mathrm{m}$.

Without loss of generality we may assume $p=0$, i.e., $\mathscr{D}^{\circ}(\mathscr{R})$ is a normalized dualizing complex. By [GW2, Theorem 2.1.4], it is known that $\underline{\operatorname{Hom}}_{k}(\cdot, \ell)$ is a dualizing functor on the category of $\mathbf{Z}^{n}$-graded $\mathscr{R}$-modules. Now let $\underline{\vartheta} \mathscr{R}$ be as above a homogeneous ideal with $\operatorname{Rad} \underline{\vartheta} \mathscr{R}=m$.

4.2. Proposition. $\mathscr{D}^{\circ}(\mathscr{R}):=\underline{\operatorname{Hom}}_{\mathscr{A}}\left(\mathscr{K}^{\circ}(\underline{\vartheta} ; \mathscr{R}), h\right)$ is a normalized dualizing complex of $\mathscr{R}$.

Proof. Obviously $\mathscr{D}^{*}(\mathscr{R})$ is a bounded complex of $\mathbf{Z}^{n}$-graded $\mathscr{R}$-modules and homomorphisms. As a direct sum of localizations $\mathscr{K}^{i}(\underline{\vartheta} ; \mathscr{R}), i \in \mathbf{Z}$, is a flat $\mathscr{R}$-module. Therefore, $\mathscr{D}^{-i}(\mathscr{R}), i \in \mathbf{Z}$, is a $\mathbf{Z}^{n}$-graded injective $\mathscr{R}$-module. Because $H^{i}\left(\mathscr{K}^{*}(\underline{\vartheta} ; \mathscr{R})\right)$ is an Artinian $\mathbf{Z}^{n}$-graded $\mathscr{R}$-module, it follows by duality that $H^{-i}\left(\mathscr{Z}^{\circ}(\mathscr{R})\right)$ is a finitely generated $\mathbf{Z}^{n}$-graded $\mathscr{R}$-module. Because of

$$
\underline{\operatorname{Hom}}_{\mathscr{R}}\left(h, \mathscr{D}^{-i}(\mathscr{R})\right) \cong \operatorname{Hom}_{h}\left(\not h \otimes \mathscr{K}^{i}(\underline{\vartheta} ; \mathscr{R}), h\right)= \begin{cases}h, & i=0, \\ 0, & i \neq 0,\end{cases}
$$

the claim follows.

Now let us construct the dualizing complex for the particular case of an affine semigroup ring $\ell[S]$. To this end, put $\underline{Y}=\left\{\underline{x}^{\underline{s}} \mid \underline{s} \in E_{0}(S)\right\}$. We denote by $r$ the finite number of elements of $\underline{Y}$. Then $\operatorname{Max}_{S}=\left\{J_{1}, \ldots, J_{r}\right\}$ by virtue of Remark 1.6. By Lemma $3.3 \underline{Y}$ generates an $m$-primary ideal in $k[S]$. Let $\mathscr{K}^{*}(\underline{Y} ; \not[S])$ denote the complex of $\mathbf{Z}^{n}$-graded $h[S]$-modules, as constructed 
at the beginning of this section with respect to the system $\underline{Y}$ of elements of $k[S]$. Then there is a $\mathbf{Z}^{n}$-graded isomorphism $H_{m}^{i}(\ell[S]) \cong H^{i}(Y ; k[S])$, $i \in \mathbf{Z}$, where we write $H^{i}(\underline{Y} ; \not[S])$ instead of $\left.H^{i}\left(\mathscr{K}^{*}(\underline{Y} ; \not / S]\right)\right)$. At first we describe $\mathscr{K}^{*}(\underline{Y} ; \not[S])$ more precisely in terms of the semigroup $S$.

\subsection{Lemma. It holds}

$$
\left.\mathscr{K}^{i}(\underline{Y} ; \not)[S]\right)= \begin{cases}0, & i<0 \text { or } i>r, \\ k[S], & i=0, \\ \bigoplus_{1 \leq j_{1}<\cdots<j_{i} \leq r} \not\left[S-F_{\cap_{l=1}^{i} J_{j_{l}}}\right], & 0<i \leq r .\end{cases}
$$

Proof. By virtue of the construction of $\mathscr{K}^{\circ}(\underline{Y} ; \not[S])$ the conclusion follows because of Theorem 1.7 and properties of the localization.

Let $\mathscr{K}^{\circ}(\underline{Y} ; k[S])_{\underline{s}}, \underline{s} \in \mathbf{Z}^{n}$, denote the complex, which is the restriction of $\mathscr{K}^{\circ}(\underline{Y} ; \not[S])$ to its sth graded piece. It is a subcomplex of $\mathscr{K}^{*}(\underline{Y} ; \not[S])$ such that

$$
\begin{aligned}
H^{i}(\underline{Y} ; \not l[S]) & \left.=\bigoplus_{\underline{s} \in \mathbf{Z}^{n}} H^{i}\left(\mathscr{K}^{\cdot}(\underline{Y} ; \not l S]\right)_{\underline{s}}\right) \\
& =\bigoplus_{\underline{s} \in G(S)} H^{i}\left(\mathscr{K}^{\cdot}(\underline{Y} ; \not[S])_{\underline{s}}\right), \quad i \in \mathbf{Z},
\end{aligned}
$$

since $\mathscr{K}^{i}(\underline{Y} ; \not[S])_{s}=0$ for $\underline{s} \notin G(S)$. Let $\mathscr{U}$ be a filter of $\mathscr{F}_{S}$. With the notation $\mathscr{F}_{S} / \mathscr{U}, S_{\mathscr{U}}, \Delta, \Delta_{\mathscr{U}}$ as defined in $\S 2$, we prove

4.4. Lemma. Let $\underline{s} \in S_{\mathscr{U}}$. Then $\left.\mathscr{K}^{*}(\underline{Y} ; \not l S]\right)_{\underline{s}} \cong \mathscr{K}^{*}\left(\Delta, \Delta_{\mathscr{U}} ; k\right)[1]$, where the complex on the right side is the relative cochain complex of $\Delta$ with respect to $\Delta_{\mathscr{U}}$ shifted one place to the right.

Proof. Let $J_{j_{1}}, \ldots, J_{j_{i}} \in \operatorname{Max}_{S}$. Then

$$
\mathscr{f}\left[S-F_{J_{j_{1}} \cap \cdots \cap J_{j_{i}}}\right]_{\underline{s}}= \begin{cases}\mathscr{L}, & J_{j_{1}} \cap \cdots \cap J_{j_{i}} \in \mathscr{U}, \\ 0, & \text { otherwise. }\end{cases}
$$

By definition $J_{j_{1}} \cap \cdots \cap J_{j_{i}} \in \mathscr{U}$ if and only if $\left\{j_{1}, \ldots, j_{i}\right\} \in \Delta \backslash \Delta_{\mathscr{U}}$. Whence the analogous building of the complexes yields the claim.

With Lemma 4.4 in mind one may calculate the cohomology of the complex $\mathscr{K}^{*}(\underline{Y} ; \not k[S])$.

4.5. Lemma. For $i \in \mathbf{Z}$ there are $\mathbf{Z}^{n}$-graded isomorphisms of $k$-vector spaces

$$
H^{i}(\underline{Y} ; \not[S]) \cong \bigoplus_{\mathscr{U} C \mathscr{F}_{S}} \ell\left[S_{\mathscr{U}}\right] \otimes_{k} H_{i-2}\left(\mathscr{F}_{S} / \mathscr{U} ; \not\right),
$$

where the direct sum is taken over all filters $\mathscr{U}$ of $\mathscr{F}_{S}$.

Proof. Because $\Delta$ has trivial cohomology groups, the following exact sequences of complexes

$$
0 \rightarrow \mathscr{K}^{*}\left(\Delta, \Delta_{\mathscr{U}} ; \ell\right) \rightarrow \mathscr{K}^{*}(\Delta ; \not) \rightarrow \mathscr{K}^{*}\left(\Delta_{\mathscr{U}} ; \not k\right) \rightarrow 0
$$


yield isomorphisms

$$
H^{i-1}\left(\Delta, \Delta_{\mathscr{U}} ; \ell\right) \cong \widetilde{H}^{i-2}\left(\Delta_{\mathscr{U}} ; \ell\right), \quad i \in \mathbf{Z} .
$$

Because $h$ is a field,

$$
\widetilde{H}^{i-2}\left(\Delta_{\mathscr{U}} ; k\right) \cong \widetilde{H}_{i-2}\left(\Delta_{\mathscr{U}} ; k\right)
$$

by duality. By the previous considerations

$$
\begin{aligned}
H^{i}(\underline{Y} ; \not[S]) & \cong \bigoplus_{\underline{s} \in G(S)} H^{i}\left(\mathscr{K}^{\cdot}(\underline{Y} ; \not[S])_{\underline{s}}\right) \\
& \cong \bigoplus_{\mathscr{U} \subset \mathscr{F}_{S}} \bigoplus_{\underline{s} \in S_{U}} H^{i}\left(\mathscr{K}^{\cdot}(\underline{Y} ; \not l[S])_{\underline{s}}\right) .
\end{aligned}
$$

Recall that $G(S)=\bigcup_{\mathscr{U} C, F_{S}} S_{\mathscr{U}}$ and $S_{\mathscr{U}} \cap S_{\mathscr{U}^{\prime}}=\varnothing$ for $\mathscr{U} \neq \mathscr{U}^{\prime}$. Finally we have

$$
\left.H^{i}(\underline{Y} ; \not)[S]\right) \cong \bigoplus_{\mathscr{U} \subset \mathscr{F}_{S}} k\left[S_{\mathscr{U}}\right] \otimes_{\not} H^{i-1}\left(\Delta, \Delta_{\mathscr{U}} ; \not\right)
$$

by view of Lemma 4.4. With the above remark, the claim follows.

4.6. Theorem. Let $\mathscr{K}^{*}(\underline{Y} ; \not[S])$ be the above complex of $\mathbf{Z}^{n}$-graded $k[S]$ modules. Then

$$
\mathscr{D}^{\circ}(\not{k}[S])=\underline{\operatorname{Hom}}_{k}\left(\mathscr{K}^{*}(\underline{Y} ; \not k[S]), \not k\right)
$$

is the dualizing complex of $k[S]$ and

$$
H^{-i}\left(\mathscr{D}^{\circ}(k[S])\right) \cong \bigoplus_{\mathscr{U} \subset \mathscr{F}_{S}} k\left[-S_{U}\right] \otimes_{k} H_{i-2}\left(\mathscr{F}_{S} / \mathscr{U} ; k\right),
$$

$i \in \mathbf{Z}$, as $h$-vector spaces.

Proof. By construction and Proposition 4.2 $D^{\circ}(\ell[S])$ is the normalized dualizing complex of $k[S]$. Because of the dualizing functor, the claim about the cohomology follows by Lemma 4.5.

For certain reasons it is useful to know a complex of finitely generated modules quasi-isomorphic to the dualizing complex.

4.7. Corollary. The dualizing complex $D^{*}(\ell[S])$ is quasi-isomorphic to a complex of finitely generated $\mathbf{Z}^{n}$-graded $\ell[S]$-modules.

Proof. The cohomology modules of $\mathscr{K}^{\circ}(\underline{Y} ; \not[S])$ are Artinian Z-graded $k[S]$ modules. Therefore, there is an $\underline{s} \in \mathbf{Z}^{n}$ such that

$$
H^{i}(\underline{Y} ; \not h[S])_{\underline{t}}=0 \text { for all } i \in \mathbf{Z} \text { or it holds } \underline{t} \leq \underline{s}, \underline{t} \in \mathbf{Z}^{n} .
$$

Define

$$
\mathscr{E}^{\cdot}(k[S])=\bigoplus_{\substack{t \in G(S) \\ t \geq-\underline{s}}} \mathscr{D}^{\cdot}(\mathcal{k}[S])_{\underline{t}}
$$


with the induced $\ell[S]$-module structure and the induced boundary maps. As easily seen $\mathscr{E}^{\circ}(\not{k}[S])$ is a subcomplex of $\mathscr{D}^{\circ}(\not[S])$ with isomorphic cohomology modules. Without loss of generality we may choose $\underline{s} \in G(S)$. Then $\left\{\underline{t} \mid \mathscr{E}^{i}(\ell[S])_{\underline{t}} \neq 0\right.$ for some $\left.i \in \mathbf{Z}\right\} \subseteq-\underline{s}+\bar{S}$. Now there is an integer $j$, such that the dimension of every $\ell$-vector space $\mathscr{E}^{i}(\ell[S])_{t}$ is bounded above by $j$. Because $\ell[\bar{S}]$ is a finitely generated $\ell[S]$-module, the proof is complete.

\section{LOCAL COHOMOLOGY AND CANONICAL MODULE}

As an immediate consequence of the construction of $\mathscr{K}^{\circ}(\underline{Y} ; \not[S])$ and its properties we get the following result about the local cohomology modules $H_{m}^{i}(k[S]), i \in \mathbf{Z}$, of $k[S]$.

5.1. Corollary. For an $i \in \mathbf{Z}$ there is an isomorphism of $\mathbf{Z}^{n}$-graded $k$-vector spaces

$$
H_{m}^{i}(k[S]) \cong \bigoplus_{\mathscr{U} \subset \mathscr{F}_{S}} k\left[S_{\mathscr{U}}\right] \otimes_{k} H_{i-2}\left(\mathscr{F}_{S} / \mathscr{U} ; \not\right),
$$

where the sum is taken over all filters $\mathscr{U}$ of $\mathscr{F}_{S}$.

By Lemma 4.5 and the results of $\S 4$, the proof is obvious. The next remark extends this result to certain finitely generated $\mathbf{Z}^{n}$-graded $\ell[S]$-modules.

5.2. Remark. Let $\underline{t}_{1}, \ldots, \underline{t}_{l} \in \mathbf{Z}^{n}$ and $N:=\left\{\underline{t}_{1}, \ldots, \underline{t}_{l}\right\}+S$. Then $\ell[N]$ is a finitely generated $\mathbf{Z}^{n}$-graded $\ell[S]$-module. Put

$$
N_{\mathscr{U}}=\bigcap_{\mathbf{Q}^{+} F_{J} \in \mathscr{U}}\left(N-F_{J}\right) \backslash \bigcup_{\mathbf{Q}^{+} F_{l} \notin \mathscr{U}}\left(N-F_{I}\right) .
$$

Then, by the same considerations as done in $\S 4$, one obtains "mutatis mutandis"

$$
H_{m}^{i}(k[N]) \cong \bigoplus_{\mathscr{U} \subset \mathscr{F}_{S}} k\left[N_{\mathscr{U}}\right] \otimes_{k} H_{i-2}\left(\mathscr{F}_{S} / \mathscr{U} ; \not\right)
$$

for the $i$ th local cohomology module of the $k[S]$-module $k[N]$. Of a particular interest are the local cohomology modules in small dimensions.

5.3. Corollary. $H_{m}^{0}(\ell[S])=0, H_{m}^{1}(\ell[S])=\ell\left[\left(\bigcap_{J \in \mathrm{Max}_{S}}\left(S-F_{J}\right)\right) \backslash S\right]$.

Proof. We have $\widetilde{H}^{-2}\left(\Delta_{\mathscr{U}} ; \not\right)=0$ and $\widetilde{H}^{-1}\left(\Delta_{\mathscr{U}} ; \not\right)=\not{Z}$, if $\Delta_{\mathscr{U}}=\varnothing$ resp. $=0$ if $\Delta_{\mathscr{U}} \neq \varnothing$ for every filter $\mathscr{U}$ of $\mathscr{F}_{S}$. Now $\Delta_{\mathscr{U}}=\varnothing$ if and only if $\mathscr{U}=$ $\mathscr{F}_{S} \backslash\{\{\underline{0}\}\}$, i.e., $S_{\mathscr{U}}=\bigcap_{J \in \operatorname{Max}_{S}}\left(S-F_{J}\right) \backslash S$. Therefore the claim follows by Lemma 4.5.

5.4. Example. Let $d=\operatorname{dim} k[S]=2$. By the example after Remark 1.6 we get $n=2$ and $F_{S}=\left\{\{\underline{0}\}, \mathbf{Q}^{+} F_{1}, \mathbf{Q}^{+} F_{2}, \mathscr{C}_{S}\right\}$. Therefore one has to consider the following filters:

1. $\mathscr{U}_{1}=\left\{\mathscr{C}_{S}\right\}$ with $\mathscr{F}_{S} / \mathscr{U}_{1} \cong \mathscr{F}_{S}$ and $S_{\mathscr{U}_{1}}=G(S) \backslash\left(S-F_{1} \cup S-F_{2}\right)$.

2. $\mathscr{U}_{2 i}=\left\{\mathscr{C}_{S}, \mathbf{Q}^{+} F_{i}\right\}$ with $\mathscr{F}_{S} / \mathscr{U}_{2 i}=\left\{\{\underline{0}\}, \mathbf{Q}^{+} F_{j}, \mathscr{C}_{S}\right\}$ and $S_{\mathscr{U}_{2 i}}=$ $\left(S-F_{i}\right) \backslash\left(S-F_{j}\right)$ where $\{i, j\}=\{1,2\}$. 
3. $\mathscr{U}_{3}=\left\{\mathscr{C}_{S}, \mathbf{Q}^{+} F_{1}, \mathbf{Q}^{+} F_{2}\right\}$ with $\mathscr{F}_{S} / \mathscr{U}_{3} \cong\left\{\{\underline{0}\}, \mathscr{C}_{S}\right\}$ and $S_{\mathscr{U}_{3}}=$ $\left(S-F_{1}\right) \cap\left(S-F_{2}\right) \backslash S$.

Up to $H_{0}\left(\mathscr{F}_{S} / \mathscr{U}_{1} ; \not\right)=H_{-1}\left(\mathscr{F}_{S} / \mathscr{U}_{3} ; \ell\right)=k$, all of the other homologies vanish. That is,

$$
H_{m}^{i}(k[S])=\left\{\begin{array}{l}
0, \quad i \leq 0 \text { or } i>2, \\
k\left[\left(S-F_{1}\right) \cap\left(S-F_{2}\right) \backslash S\right], \quad i=1, \\
k\left[G(S) \backslash\left(\left(S-F_{1}\right) \cup\left(S-F_{2}\right)\right)\right], \quad i=2 .
\end{array}\right.
$$

Now put $S^{\prime}=\bigcap_{i=1}^{n} S-F_{i}$ as defined in Lemma 1.8. $S^{\prime}$ is bounded below. By Lemma $1.10 k\left[S^{\prime}\right]$ is a finitely generated $\mathbf{Z}^{n}$-graded $k[S]$-module. One has $S \subseteq S^{\prime} \subseteq \bar{S}, S^{\prime}+S \subseteq S^{\prime}$. Hence $S^{\prime}$ is a standard semigroup. In order to calculate the local cohomology modules of $k\left[S^{\prime}\right]$, note that $\underline{Y} k\left[S^{\prime}\right]$ is a $\mathscr{K}\left[S^{\prime} \backslash\{\underline{0}\}\right]$-primary ideal. Hence, the complex $\left.\mathscr{K}^{\circ}\left(\underline{Y} ; \not S^{\prime}\right]\right)$ can be viewed as a complex of $\ell[S]^{-}$as well as a complex of $\ell\left[S^{\prime}\right]$-modules. Let $\mathscr{U}(J)$ be defined as in Lemma 1.8. By Lemma 1.8 and Remark 5.2 it follows immediately:

5.5. Corollary. For $i \in \mathbf{Z}$ there is an isomorphism of $\mathbf{Z}^{n}$-graded $\mathfrak{k}$-vector spaces

$$
H_{m}^{i}\left(k\left[S^{\prime}\right]\right) \cong \bigoplus_{J \subseteq\{1, \ldots, n\}} k\left[S_{\mathscr{U}(J)}^{\prime}\right] \otimes_{k} H_{i-2}\left(\mathscr{F}_{S} / \mathscr{U}(J) ; k\right),
$$

where the sum is taken over all $J \subseteq\{1, \ldots, n\}$.

This result was shown by Trung and Hoa [TH, §3]. The simplification in comparison with the general case is based on $S_{\mathscr{U}}=\varnothing$ for all filters $\mathscr{U}$ not of the form $\mathscr{U}(J)$ (see Lemma 1.8).

We shall calculate now $H_{m}^{d}(\ell[S]), d=\operatorname{dim} \ell[S]$. To this end, put

$$
S^{\prime \prime}=G(S) \backslash \bigcup_{i=1}^{n}\left(F_{i}-S\right) \text {. }
$$

5.6. Theorem. There is an isomorphism of $\mathbf{Z}^{n}$-graded $\not S[S]$-modules

$$
H_{m}^{d}(\ell[S]) \cong h\left[-S^{\prime \prime}\right],
$$

$d=\operatorname{dim} \ell[S]$.

Proof. Since $H_{m}^{d}(\not[S])$ is an Artinian module, it follows by Corollary 5.1. that $S_{\mathscr{U}}$ is bounded above, provided $H_{d-2}\left(\mathscr{F}_{S} / \mathscr{U} ; \not\right) \neq 0$. It is easy to see that the sets $S_{\mathscr{U}(\{j\})}, j=1, \ldots, n$, are nonempty and not bounded above (see Lemma $1.9(\mathrm{~d}))$. Therefore,

$$
H_{d-2}\left(\mathscr{F}_{S} / \mathscr{U}_{(\{j\})} ; \not\right)=0 \text { for } j=1, \ldots, n .
$$

For two filters $\mathscr{U} \subseteq \mathscr{U}^{\prime}$ of $\mathscr{F}_{S}$ the order complex of $\mathscr{F}_{S} / \mathscr{U}^{\prime}$ may be viewed as a subcomplex of the order complex of $\mathscr{F}_{S} / \mathscr{U}$. By the aid of the Mayer-Vietoris sequence it follows:

$$
\begin{gathered}
H_{d-2}\left(\mathscr{F}_{S} / \mathscr{U}\right)=0 \text { for some } \mathscr{U} \supseteq \mathscr{U}(\{j\}) \text { and some } j=1,2, \ldots, n . \text { Hence } \\
H_{m}^{d}(\not{k}[S]) \cong \mathscr{k}\left[S_{\left\{F_{s}\right\}}\right] \otimes_{k} H_{d-2}\left(\mathscr{F}_{S} ; \not k\right),
\end{gathered}
$$


as an isomorphism of $k$-vector spaces, Because of $2.3(\mathrm{~b})$ and

$$
S_{\left\{\mathscr{F}_{S}\right\}}=G(S) \backslash \bigcup_{J \neq \varnothing} S-F_{J}
$$

there is an isomorphism of $\mathrm{Z}^{n}$-graded $\not$-vector spaces $H_{m}^{d}(\not{k}[S]) \cong \not\left[-S^{\prime \prime}\right]$. Because of the structure of the dualizing complex, this is in fact an isomorphism of $\mathbf{Z}^{n}$-graded $k[S]$-modules

$$
H_{m}^{d}(\not{k}[S]) \cong \not k[G(S)] / \not{k}\left[\bigcup_{j=1}^{n} S-F_{j}\right] .
$$

The $d$ th local cohomology module of $k[S]$ is closely related to the canonical module of $k[S]$. Here

$$
K_{\not L[S]}=H^{-d}\left(\mathscr{D}^{\circ}(\ell[S])\right), \quad d=\operatorname{dim} \ell[S],
$$

is called the canonical module of $k[S]$. See Herzog and Kunz [HK], resp. Schenzel $[\mathrm{Sch}]$ for the definition and basic results.

5.7. Corollary. There are isomorphisms of $\mathbf{Z}^{n}$-graded $\ell[S]$-modules

$$
K_{k[S]} \cong k\left[S^{\prime \prime}\right] \cong K_{\ell\left[S^{\prime}\right]} .
$$

Proof. Duality yields the first isomorphism by Theorem 5.6. By the same argument and Lemma 1.8, we get the second isomorphism.

We shall conclude this section with the calculation of the local cohomology modules of the canonical module of $\ell[S]$. This is useful in order to simplify a Cohen-Macaulay criterion.

5.8. Theorem. For $i \in \mathbf{Z}$ it holds

$$
H_{m}^{i}\left(K_{\ell[S]}\right) \cong \bigoplus_{J \subseteq\{1, \ldots, n\}} k\left[-S_{\mathscr{U}(\{1, \ldots, n\} \backslash J\}}^{\prime}\right] \otimes_{k} H_{i-2}\left(\mathscr{F}_{S} / \mathscr{U}_{J} ; \not\right) .
$$

Proof. First note that $K_{\ell[S]}$ is a finitely generated $\mathbf{Z}^{n}$-graded $\ell[S]$-module. Furthermore $S^{\prime \prime}+S^{\prime} \subseteq S^{\prime \prime}$. Thus, we may apply Remark 5.2 in order to claim. It is enough to prove that

$$
S_{\mathscr{U}(J)}^{\prime \prime}=-S_{\mathscr{U}(\{1, \ldots, n\} \backslash J)}^{\prime}
$$

for $J \subseteq\{1, \ldots, n\}$. But

$$
\begin{aligned}
S_{\mathscr{U}(J)}^{\prime \prime} & =\bigcap_{j \in J}\left(S^{\prime \prime}-F_{j}\right) \backslash \bigcup_{i \notin J}\left(S^{\prime \prime}-F_{i}\right) \\
& =\bigcap_{j \in J}\left(G(S) \backslash\left(F_{j}-S\right)\right) \backslash \bigcup_{i \notin J}\left(G(S) \backslash\left(F_{i}-S\right)\right)
\end{aligned}
$$

as follows by simple calculations. This proves the statement. 


\section{Applications}

This section is devoted the study of homological properties of affine semigroup rings. To this end we relate the fine structure of the dualizing complex to structural properties of these rings. Let $\mathscr{R}$ denote a Noetherian $\mathbf{N}^{n}$-graded ring with $\mathscr{R}_{0}=k$ and $m:=\bigoplus_{\underline{s} \neq \underline{0}} \mathscr{R}_{\underline{s}}$. The complex $\tau^{-d} \mathscr{D}(\mathscr{R}), d=\operatorname{dim} \mathscr{R}$, denotes the left truncated dualizing complex $\mathscr{D}^{\circ}(\mathscr{R})$ of $\mathscr{R}$, i.e.,

$$
0 \rightarrow \operatorname{im}\left(\mathscr{D}^{-d}(\mathscr{R}) \rightarrow \mathscr{D}^{-d+1}(\mathscr{R})\right) \rightarrow \mathscr{D}^{-d+1}(\mathscr{R}) \rightarrow \cdots
$$

with the induced homomorphisms. For the cohomology modules it yields

$$
H^{i}\left(\tau^{-d} \mathscr{D}^{\cdot}(\mathscr{R})\right) \cong\left\{\begin{array}{l}
H^{i}\left(\mathscr{D}^{\cdot}(\mathscr{R})\right), \quad-d<1 \leq 0, \\
0, \quad \text { otherwise. }
\end{array}\right.
$$

As before, let $K_{\mathscr{R}}$ denote the canonical module of $\mathscr{R}$. We need the following criterion.

6.1. Theorem. The ring $\mathscr{R}$ is

- locally a Cohen-Macaulay ring (i.e., $\mathscr{R}_{n}, \rho \subset m$ a prime ideal, is a Cohen-Macaulay ring) if and only if $H^{i}\left(\tau^{-d} \mathscr{D}(\mathscr{R})\right), i \in \mathbf{Z}$, are $\mathscr{R}$ modules of finite length.

- a Buchsbaum ring if and only if $\tau^{-d} \mathscr{D}^{\circ}(\mathscr{R})$ is quasi-isomorphic to a complex of $\mathscr{R} \mid m$-vector spaces.

- a Cohen-Macaulay ring if and only if $H^{i}\left(\tau^{-d} \mathscr{D}^{*}(\mathscr{R})\right)=0$ for all $i \in \mathbf{Z}$.

- a Gorenstein ring if and only if it is a Cohen-Macaulay ring with $\mathscr{R} \cong$ $K_{\mathscr{A}}(\underline{s})$ for some $\underline{\underline{s}} \in \mathbf{Z}^{n}$.

- $\mathscr{\mathscr { S }}_{1}$, i.e., depth $\mathscr{R}_{\mu} \geq \operatorname{Min}\left\{l, \operatorname{dim} \mathscr{R}_{\mu}\right\}$ for all prime ideals $\mu$ of $\mathscr{R}$, $l \geq 2$, if and only if $\mathscr{R}$ is $\mathscr{S}_{2}$ and $H_{m}^{i}\left(K_{.}\right)=0$ for all $i, d-l+2 \leq$ $i<d$.

In the case $\mathscr{R}$ is locally a Cohen-Macaulay ring with $d=\operatorname{dim} \mathscr{R} \geq 2$, there are a short exact sequence

$$
0 \rightarrow H^{-1}(\mathscr{D}(\mathscr{R})) \rightarrow H_{m}^{d}\left(K_{, \mathscr{R}}\right) \rightarrow \underline{\operatorname{Hom}}_{\not}(\mathscr{R}, \not h) \rightarrow 0
$$

and isomorphisms

$$
H_{m}^{d+1-j}\left(K_{\mathscr{R}}\right) \cong H^{-j}\left(\mathscr{D}^{\cdot}(\mathscr{R})\right), \quad 2 \leq j<d,
$$

of $\mathbf{Z}^{n}$-graded $\mathscr{R}$-modules.

The proofs are similar to those of the local situation given in [Sch]. In the following, let $\mathscr{R}=k[S]$, an affine semigroup ring.

A. The Serre condition $\mathscr{S}_{1}$. Let $\operatorname{dim} \ell[S]=d \geq 2$. First note that $\mathscr{S}_{1}$ is always satisfied because $\not R[S]$ is a domain. Moreover $\mathscr{S}_{d}$ is equivalent to the Cohen-Macaulayness of $\mathscr{k}[S]$. Thus, in a certain sense $\mathscr{S}_{l}$ measures the Cohen-Macaulay deviation. 
6.2. Lemma. The ring $k[S]$ satisfies $\mathscr{S}_{l}, l \geq 2$, if and only if

$$
\operatorname{depth} \ell\left[S-F_{J}\right] \geq \operatorname{Min}\left\{l, \operatorname{dim} \ell\left[S-F_{J}\right]\right\}
$$

for all $J$ such that $\varnothing \neq J \subseteq\{1, \ldots, n\}$.

Proof. The claim says that $\mathscr{S}_{l}$ holds if and only if

$$
\operatorname{depth} \ell[S]_{(\mu)} \geq \operatorname{Min}\left\{1, \operatorname{dim} \ell[S]_{(\mu)}\right\}
$$

for all homogeneous prime ideals $\rho$ of $k[S]$ (see Theorem 3.2). But this equivalence is shown by Goto and Watanabe [GW2, p. 244].

6.3. Theorem. Let $S^{\prime}=\bigcap_{i=1}^{n} S-F_{i}$ as above. Then $k[S]$ satisfies $\mathscr{S}_{2}$ if and only if $S=S^{\prime}$.

Proof. First let $S=S^{\prime}$. Then $H_{m}^{1}(\ell[S])=0$ by Corollary 5.3. Let $J \subseteq$ $\{1, \ldots, n\}$ be a subset with at least two elements. Then $S-F_{J}=\bigcap_{j \in J} S-$ $F_{J}-F_{j}$. By the same argument as before

$$
H_{\ell\left[\left(S \backslash F_{J}\right)+S-F_{J}\right]}^{1}\left(\not \subset\left[S-F_{J}\right]\right)=0 .
$$

By Lemma 6.2 this means nothing else but $\mathscr{S}_{2}$.

In order to prove the converse statement, let $S \neq S^{\prime}$ and $\underline{t} \in S^{\prime} \backslash S$. Put $I_{\underline{t}}:=$ $\{\underline{s} \in S \mid \underline{s}+\underline{t} \in S\}$. It follows $I_{\underline{t}}+S \subseteq I_{\underline{t}}$ and $I_{\underline{t}} \cap F_{i} \neq \varnothing$ for all $i \in\{1, \ldots, n\}$. Therefore, $k\left[I_{\underline{t}}\right]$ is a homogeneous ideal of $\not{k}[S]$, not contained in one of the $\ell\left[S \backslash F_{i}\right]$, the homogeneous prime ideals of height 1 of $\ell[S]$. Hence the height of $k\left[I_{\underline{t}}\right]$ is at least two. Because of the $\mathscr{S}_{2}$-condition it contains a $k[S]$-regular sequence $\left\{v_{1}, v_{2}\right\}$. Whence

$$
v_{1} v_{2} \underline{x}^{\underline{t}} \in v_{1} k[S] \text { and } v_{1} \underline{x}^{\underline{t}} \in v_{1} \ell[S]: v_{2}=v_{1} k[S] \text {, }
$$

i.e., $\underline{t} \in S$, a contradiction.

6.4. Theorem. Let $l \geq 2$ be an integer. Then $k[S]$ satisfies $\mathscr{S}_{l}$ if and only if the following holds: $S^{\prime}=S$ and for any subset $J, \varnothing \neq J \subset\{1,2, \ldots, n\}$, $S_{\mathscr{U}(\{1, \ldots, n\} \backslash J)}^{\prime}=\varnothing$ or $H_{i}\left(\mathscr{F}_{S} / \mathscr{U}(J) ; \ell\right)=0$ for all $i \geq d-l$.

Proof. By virtue of Example 2.3, Theorem 5.6, and Theorem 5.8 the claim results immediately from Theorem 6.1 .

B. When is $k[S]$ locally a Cohen-Macaulay ring? The understanding of the relationship between $k[S]$ and $k\left[S^{\prime}\right]$ becomes more significant by the following statement.

6.5. Lemma. Assume $\ell[S]$ is locally a Cohen-Macaulay ring. Then the following conditions are equivalent:

(i) $S^{\prime} \backslash S$ is a finite set, and

(ii) $S^{\prime}=\bigcap_{J \in \operatorname{Max}_{S}} S-F_{J}$. 
Under one of these conditions $H_{\prime \prime \prime}^{i}(\ell[S])=H_{\prime \prime \prime}^{i}\left(\ell\left[S^{\prime}\right]\right)$ for all $i \geq 2$.

Proof. First note that (ii) yields (i) by Corollary 5.3 and the short exact sequence given in Theorem 6.1. Now consider the short exact sequence

$$
0 \rightarrow \not[S] \rightarrow \not\left[S^{\prime}\right] \rightarrow \not\left[S^{\prime} \backslash S\right] \rightarrow 0 .
$$

Under the assumption (i) it gives isomorphisms

$$
\ell\left[S^{\prime} \backslash S\right] \cong H_{m}^{0}\left(\ell\left[S^{\prime} \backslash S\right]\right) \cong H_{m}^{1}(\ell[S]) \cong \ell\left[\bigcap_{J \in \operatorname{Max}_{S}}\left(S-F_{J}\right) \backslash S\right]
$$

(see Corollary 5.3). Therefore (ii) holds. Now the isomorphisms follow by the short exact sequence.

Now let $r(J), J$ being a subset of $\{1, \ldots, n\}$, defined as in Lemma 1.4. Then there is the following criterion for $k[S]$ being locally a Cohen-Macaulay ring.

6.6. Theorem. The following conditions are equivalent:

(i) $k[S]$ is locally a Cohen-Macaulay ring.

(ii) $S^{\prime}=\bigcap_{J \in \operatorname{Max}_{S}} S-F_{J}$ and $k\left[S^{\prime}\right]$ is locally a Cohen-Macaulay ring.

(iii) $S^{\prime} \backslash S$ is a finite set and

$$
S_{\mathscr{U}(J)}^{\prime}=\varnothing \quad \text { or } H_{i}\left(\mathscr{F}_{S} / \mathscr{U}(J) ; \not\right)=0 \quad \text { for all } i \in \mathbf{Z}
$$

and every subset of $J$ of $\{1, \ldots, n\}$ with $r(J) \neq\{1, \ldots, n\}$.

(iv) For every subset $J$ of $\{1, \ldots, n\}$ with $r(J) \neq\{1, \ldots, n\}, \ell\left[S-F_{J}\right]$ is a Cohen-Macaulay ring.

Proof. The equivalence of (i) and (ii) follows by Theorem 6.1 and Lemma 6.5. By virtue of Theorem 4.6 the equivalence of (ii) and (iii) turns out by Theorem 6.1 and Lemma 6.5. Finally looking at the corresponding localizations, Theorem 4.6 proves the equivalence of (iii) and (iv).

6.7. Examples. (i) Suppose $r(J)=J$ for every subset $J$ of $\{1, \ldots, n\}$ with $r(J) \neq\{1, \ldots, n\}$; e.g., this is the case when rank $G(S) \leq 3$ of $S$ is a simplicial semigroup (see Remark 1.6 or Example 1.11 ). Then $k[S]$ is locally a CohenMacaulay ring if and only if $S^{\prime} \backslash S$ is a finite set (see Lemma 6.5).

(ii) Let $S$ be an affine standard semigroup such that $k[S]$ is locally a CohenMacaulay ring. Let $U$ denote a finite subset of $S \backslash\{\underline{0}\}$ with the following property: If $\underline{s}+\underline{t} \in U$ for some $\underline{s}, \underline{t} \in S \backslash\{\underline{0}\}$, then $\underline{s}, \underline{t} \in U$. Then $S_{1}=S^{\prime} \backslash U$ is a standard semigroup such that $S_{1}^{\prime}=S^{\prime}$ and $k\left[S_{1}\right]$ is locally a CohenMacaulay ring.

C. Buchsbaum rings. For $k[S]$ being a Buchsbaum ring it is necessary (but not sufficient) that $m H_{m}^{i}(\not[S])=0$ for all $i \neq \operatorname{dim} k[S]$. First let us clarify what it means for the semigroup $S$. 
6.8. Theorem. Let $k[S]$ be a Buchsbaum ring. Then $S^{\prime}+(S \backslash\{\underline{0}\}) \subseteq S$ and $H_{i}\left(\mathscr{F}_{S} / \mathscr{U}(J) ; k\right)=0$ for all $i \in \mathbf{Z}$ or $\left(S_{\mathscr{U}(J)}^{\prime}+(S \backslash\{\underline{0}\})\right) \cap S_{\mathscr{U}(J)}^{\prime}=\varnothing$ for every subset $J, \varnothing \neq J \subseteq\{1, \ldots, n\}$.

Proof. By the local duality theorem and Lemma 4.3, the claim follows from $m H_{m}^{i}(k[S])=0$ for all $i, i \neq \operatorname{dim} k[S]$, and Theorem 6.6.

6.9. Proposition. $k\left[S^{\prime}\right]$ is a Buchsbaum ring, provided $k[S]$ is a Buchsbaum ring.

Proof. If $k[S]$ is a Buchsbaum ring, then $\tau^{-d} \mathscr{D}^{*}(k[S])$ is quasi-isomorphic to a complex of $\not[S] \mid m$-vector spaces (see Theorem 6.1). Therefore, the same holds for $\tau_{-1}^{-d} \mathscr{D}^{\circ}(\ell[S])$, the complex $\tau^{-d} \mathscr{D}^{*}(\ell[S])$ truncated at $(-1)$ th place from the right. Because $S^{\prime} \backslash S$ is a finite set, $\left.H^{-1}\left(\mathscr{D}^{\circ}\left(\not S^{\prime}\right]\right)\right)=0$ and

$$
\tau_{-1}^{-d} \mathscr{D}^{\cdot}(\not[S])=\tau_{-1}^{-d} \mathscr{D}^{\cdot}\left(\mathcal{k}\left[S^{\prime}\right]\right)=\tau^{-d} \mathscr{D}^{\cdot}\left(k\left[S^{\prime}\right]\right) .
$$

Therefore $\tau^{-d} \mathscr{D}^{\circ}\left(k\left[S^{\prime}\right]\right)$ is quasi-isomorphic to a complex of $k[S] \mid m$-vector spaces, i.e., $\mathscr{L}\left[S^{\prime}\right]$ is a Buchsbaum ring (see Theorem 6.1).

The following result is a sufficient criterion. It generalizes a known result for $\mathrm{Z}$-graded rings to the $\mathbf{Z}^{n}$-graded situation.

6.10. Theorem. Put

$$
T:=\left\{\underline{t} \in \mathbf{Z}^{n} \mid H^{-i}\left(\mathscr{D}^{\cdot}(\not{R}[S])\right)_{\underline{t}} \neq 0 \text { for some } i, 0 \leq i<d\right\} .
$$

If $(T+(S \backslash\{\underline{0}\})) \cap T=\varnothing$, then $k[S]$ is a Buchsbaum ring.

Proof. For $\underline{t} \in \mathbf{Z}^{n}$ is the complex $\left(\tau^{-d} \mathscr{D}^{*}(h[S])\right)_{t}$, a complex of finite $k$-vector spaces, quasi-isomorphic to its complex of cohomology modules (with trivial boundary maps). The direct sum of these quasi-isomorphisms possessesunder the assumptions of the theorem-a trivial structure as homomorphisms of $k[S]$-modules. In particular it ensures that $m H^{-i}\left(\mathscr{D}{ }^{\circ}(k[S])\right)=0$ for all $i$, $i<d$. By Theorem 6.1 it follows that $\ell[S]$ is a Buchsbaum ring.

6.11. Examples. (i) Let $k\left[S^{\prime}\right]$ be a Cohen-Macaulay ring. Then $k[S]$ is a Buchsbaum ring if and only if $S^{\prime}+(S \backslash\{\underline{0}\}) \subseteq S$.

(ii) Let $\mathcal{J}$ denote the set of all proper nonempty subsets $J$ of $\{1, \ldots, n\}$, such that $H_{i}\left(\mathscr{F}_{S} / \mathscr{U}(J) ; k\right) \neq 0$ for some $i \in \mathbf{Z}$. Then $k\left[S^{\prime}\right]$ is a Buchsbaum ring, provided

$$
\bigcup_{J \in \mathcal{J}}\left(S_{\mathscr{U}(J)}^{\prime}+(S \backslash\{\underline{0}\})\right) \cap \bigcup_{J \in \mathscr{J}} S_{\mathscr{U}(J)}^{\prime}=\varnothing .
$$

A similar result holds also for $S$ instead of $S^{\prime}$.

D. When is $\ell[S]$ a Cohen-Macaulay ring? In order to avoid trivial situations, let $d=\operatorname{dim} \ell[S] \geq 2$. First of all, let us deduce a result shown by Hoa and Trung [TH, Theorem 4.1]. 
6.12. Proposition. $h[S]$ is a Cohen-Macaulay ring if and only if $S=S^{\prime}$ and for every nonempty proper subset $J$ of $\{1, \ldots, n\}$

$$
S_{\mathscr{U}(J)}^{\prime}=\varnothing \quad \text { or } H_{i}\left(\mathscr{F}_{S} / \mathscr{U}(J) ; \not\right)=0 \quad \text { for all } i \in \mathbf{Z} \text {. }
$$

Proof. By Theorem 6.1 the proof follows in accordance with Theorem 4.6 and Corollary 5.5.

The following criterion yields a sharpening of Proposition 6.12 based on a finer study of the dualizing complex. To this end, let $t$ be the largest integer with $2 t+2 \leq d$.

6.13. Theorem. $k[S]$ is a Cohen-Macaulay ring if and only if $S=S^{\prime}$ and for every nonempty proper subset $J$ of $\{1, \ldots, n\}$

$$
\begin{gathered}
S_{\mathscr{U}(J)}^{\prime} \cup S_{\mathscr{U}(\{1, \ldots, n\} \backslash J)}^{\prime}=\varnothing \text { or } \\
H_{i}\left(\mathscr{F}_{S} / \mathscr{U}(J) ; k\right)=0 \text { for all } i, \quad t \leq i \leq d-3 .
\end{gathered}
$$

Proof. By Theorem 6.4 it follows that $\ell[S]$ satisfies the $\mathscr{S}_{1}$-condition for $l=$ $t+2$ under the assumptions of the theorem. On the other side it yields

$$
H^{-i}\left(\mathscr{D}^{\cdot}(\not L[S])\right)=0 \text { for } t+2 \leq i<d
$$

by Theorem 4.6 and Corollary 5.5. Both of these are necessary and sufficient for $k[S]$ to be a Cohen-Macaulay ring.

6.14. Examples. (i) Let $S$ be a simplicial affine semigroup. By Lemma 1.9 $k[S]$ is a Cohen-Macaulay ring if and only if $S=S^{\prime}$. This is the well-known result shown by Goto, Suzuki,and Watanabe [GSW], resp. Stanley [St2].

(ii) Let $S=\bar{S}, \bar{S}$ is defined as in $\S 1$. That is, $S$ is a normal affine semigroup. In particular it yields $S=S^{\prime}$. Furthermore, $S_{\mathscr{U}(J)}^{\prime}=\left\{\underline{s} \in G(S) \mid s_{i} \geq 0\right.$ if and only if $i \in J\}, J$, a nonempty subset of $\{1, \ldots, n\}$, is either empty or not bounded above. Because the cohomology modules of $\mathscr{D}^{\prime}(\ell[S])$ are finitely generated, $\ell[S]$ is a Cohen-Macaulay ring (see Theorem 4.6). This was shown by Hochster [Hol] and in [KKMS].

E. Gorenstein rings. In this subsection there is a characterization of Gorenstein affine semigroup rings. To this end let

$$
S^{\prime \prime}=G(S) \backslash \bigcup_{i=1}^{n}\left(F_{i}-S\right)
$$

as in $\S 5$. The following result is shown in $[\mathrm{TH}]$.

6.15. Proposition. $k[S]$ is a Gorenstein ring if and only if $k[S]$ is a CohenMacaulay ring and there is an $\underline{s} \in G(S)$ with $S^{\prime \prime}+\{\underline{s}\}=S$.

Proof. By Corollary $\left.5.7 \not S^{\prime \prime}\right]$ is the canonical module of $k[S]$. Now $k[S]$ is a Gorenstein ring if and only if it is a Cohen-Macaulay ring with $\ell\left[S^{\prime \prime}\right]$, a monogen $\ell[S]$-module. But this is equivalent to the given condition. Furthermore, note that $S^{\prime \prime}+\{\underline{s}\}=S$ yields $S=S^{\prime}$. 
6.16. Example. Suppose $S$ is a normal affine semigroup, i.e., $S=\bar{S}$. Then $S^{\prime \prime}=S \backslash\left\{\underline{t} \in S \mid t_{i}=0\right.$ for some $\left.i \in\{1, \ldots, n\}\right\}$ as easily seen. Hence $k[S]$ is a Gorenstein ring if and only if $(1,1, \ldots, 1) \in S$.

More general in the situation of $S=\bar{S}$, one may calculate the CohenMacaulay type of $k[S]$, i.e., the minimal number of generators of the canonical module of $k[S]$. In general, this is not so easy to do. In the case of monomial space curves in $\mathbf{P}^{3}$, this is done in [BSS].

Acknowledgment. We are grateful to the referee who drew our attention to the work of Masa-Nori Ishida.

\section{REFERENCES}

[BSS] H. Bresinsky, U. Schäfer and P. Schenzel, On the canonical module of monomial curves, Comm. Algebra 15 (1987), 1799-1814.

[Br] A. Brønsted, An introduction to convex polytopes, Springer, Berlin-Heidelberg-New York, 1983.

[DI] L. E. Dickson, Finiteness of the odd perfect and primitive abundant numbers with $n$ distinct prime factors, Amer. J. Math. 35 (1913), 413-422.

[F] J. Folkman, The homology groups of a lattice, J. Math. Mech. 15 (1966), 631-636.

[GSW] S. Goto, N. Suzuki and K. Watanabe, On affine semigroup rings, Japan J. Math. 2 (1976), $1-12$.

[GW1] S. Goto and K. Watanabe, On graded rings. I, J. Math. Soc. Japan 30 (1978), 172-213.

[GW2] __, On graded rings. II. ( $\mathbf{Z}^{n}$-graded rings), Tokyo J. Math. 2 (1976), 237-261.

[Gb] W. Gröbner, Über Veronesesche Varietäten und deren Projektionen, Arch. Math. 16 (1965), 257-264.

[Gr] A. Grothendieck, Local cohomology (notes by R. Hartshorne), Lecture Notes in Math., vol. 41, Springer-Verlag, Berlin-New York, 1967.

[Ha] R. Hartshorne, Residues and duality, Lecture Notes in Math., vol. 20, Springer-Verlag, Berlin-New York, 1966.

[HK] J. Herzog and E. Kunz, Der kanonische Modul eines Cohen-Macaulay-Rings, Lecture Notes Math., vol. 238, Springer-Verlag, Berlin-New York, 1971.

[Hol] M. Hochester, Rings of invariants of tori, Cohen-Macaulay rings generated by monomials, and polytopes, Ann. of Math. 96 (1972), 318-337.

[Ho2l__, Cohen-Macaulay rings, combinatorics, and simplicial complexes, Proc. Oklahoma Ring Theory Conference, Lecture Notes in Pure and Appl. Math., vol. 26, Marcel Dekker, New York, 1977, pp. 171-223.

[I1] M.-N. Ishida, Torus embeddings and dualizing complexes, Tôhoku Math. J. 32 (1980), 111-146.

[I2] - The local cohomology groups of an affine semigroup ring, Commutative Algebra and Algebraic Geometry, in Honor of M. Nagata, Kinokuniya, Tokyo, 1988, pp. 209-221.

[KKMS] G. Kempf, F. Knudsen, D. Mumford and B. Saint-Donat, Torodial embeddings, Lecture Notes in Math., vol. 339, Springer-Verlag, Berlin-New York, 1973.

[Ku] E. Kunz, Einführung in die kommutative Algebra und algebraische Geometrie, Viewig, Braunschweig-Wiesbaden, 1980.

[Sf] U. Schäfer, Vollständige Durchschnitte unter den affinen Halgruppenringen, in preparation.

[Sch] P. Schenzel, Dualisierende Komplexe in der lokalen Algebra und Buchsbaumringe, Lecture Notes in Math., vol. 907, Springer-Verlag, Berlin-New York, 1982.

[Se] J.-P. Serre, Algèbre locale-Multiplicitès, Lecture Notes in Math., vol. 11, Springer-Verlag, Berlin-New York, 1965. 
[St1] R. P. Stanley, Hilbert functions of graded algebras, Adv. in Math. 28 (1978), 57-83.

[St2] _ Combinatorics and commutative algebra, Boston, Mass., 1983.

[St3] __ Linear diophantine equations a'ld local cohomology, Invent. Math. 68 (1982), 175193.

[SV] J. Stückrad and W. Vogel, Buchsbaum rings and applications, Springer, Berlin, 1986.

[TH] N. V. Trung and L. T. Hoa, Affine semigroups and Cohen-Macaulay rings generated by monomials, Trans. Amer. Math. Soc. 298 (1986), 145-167.

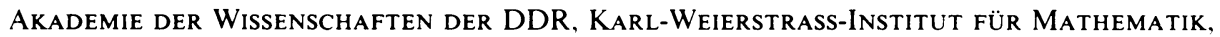
POSTFACH 1304, BERLIN, DDR-1086

Sektion Mathematik der Martin-Luther-Universität, Halle-Wittenberg, Postfach, HALLE, DDR-4010 